\title{
TROJANS IN STABLE CHAOTIC MOTION
}

\author{
E. PILAT-LOHINGER, R. DVORAK AND CH. BURGER \\ Institut fur Astronomie, Universitat Wien, \\ Türkenschanzstraße 17, A-1180 Vienna, Austria
}

\begin{abstract}
The orbits of 13 Trojan asteroids have been calculated numerically in the model of the outer solar system for a time interval of 100 million years. For these asteroids Milani et al. (1997) determined Lyapunov times less than 100000 years and introduced the notion "asteroids in stable chaotic motion". We studied the dynamical behavior of these Trojan asteroids (except the asteroid Thersites which escaped after 26 million years) within 11 time intervals - i.e. subintervals of the whole time - by means of: (1) a numerical frequency analysis (2) the root mean square (r.m.s.) of the orbital elements and (3) the proper elements. For each time interval we compared the root mean squares of the orbital elements ( $a$, e and $i)$ with the corresponding proper element. It turned out that the variations of the proper elements $e_{p}$ in the different time intervals are correlated with the corresponding r.m.s.(e); this is not the case for $\sin I_{p}$ with r.m.s.(i).
\end{abstract}

Key words: Trojan asteroids - long term evolution - proper elements

\section{Introduction}

The Trojan asteroids librating in the vicinity of Jupiter's $L_{4}$ or $L_{5}$ point are well known examples for the Lagrangian solutions of the three-body problem. The interest in this kind of motion began only in 1906 when Max Wolf discovered the first asteroid - (588) Achilles - moving near $L_{4}$ of Jupiter's orbit. More than 400 Trojan asteroids ${ }^{1}$ are known up to now, whereof, 246 have been found in the vicinity of $L_{4}$ and only 167 near $L_{5}$. This difference in the number of Trojan asteroids around the two equilibrium points is possibly caused by long term perturbations of Saturn as it was shown by a numerical study of hypothetical Trojans (cf. Barber, 1986). Actually it was believed that these asteroids are not very numerous but nowadays several thousands are thought to exist with diameters $\geq 15 \mathrm{~km}$ (cf. Shoemaker et al., 1989).

To study the problem of the Trojans theoretically with the aid of perturbation theory one has to take into account that the inclinations of these asteroids can reach large values (up to about $40^{\circ}$ ); this makes the problem different to the study of the main belt asteroids which are mostly confined to low inclined orbits. Using simplified models several theoretical studies of the motion of the Trojans have been carried out; the most complete one has been developed by Érdi $(1984,1988,1996$, 1997). Several numerical investigations have been carried out by Schubart $\&$ Bien (1984 and 1987) and by Bien \& Schubart (1984 and 1987). Milani (1993, 1994) computed the orbits of 174 Trojans in the model of the outer solar system for $10^{6}$ years and in some cases for $5 \cdot 10^{6}$ years. In a more recent study by Levison et al. (1997) the orbits of 270 fictitious $L_{4}$ Trojans and of 36 real Trojans have been computed in the model of the outer solar system up to $10^{9}$ years and $4 \cdot 10^{9}$ years, respectively.

${ }^{1}$ These asteroids are called Trojans since all of them are named after heros of the Trojan war. 
In this paper we present the orbital evolution of a sample of 13 Trojan asteroids listed in table 1, which was taken from a paper by Milani et al. (1997). In their numerical study positive Lyapunov exponents for these asteroids were computed which indicate chaotic behavior; the Lyapunov time is less than $10^{5}$ years (column 6) in all cases. Since former computations established the dynamical stability of these orbits over a much longer time interval the notion asteroids in stable chaotic motion (ASC) was introduced by these authors ${ }^{2}$. In our computations 12 of the 13 Asteroids are stable over the time interval of 100 million years and thus are stable for more than $10^{3}$ times the Lyapunov time.

Our previous computations of all known Trojan asteroids over 10 million years showed that the Trojans in stable chaotic motion have larger variations in the semimajor axis (cf. Dvorak \& Pilat-Lohinger, 1998) and one of these asteroids - (5144) Achates - has exceptionally strong variations in the inclination. The continuation of the computations of 13 Trojans (table 1) up to $10^{8}$ years yielded to one escape orbit - i.e. (1868) Thersites. The goals of this long term study of the dynamics are as follows:

1. to study the long term evolution of the orbital elements of the 13 Trojans with short Lyapunov times

2. to compute the proper elements for selected time intervals within the 100 Million years integration

3. to compute for the same time intervals the root mean square (r.m.s.) ${ }^{3}$ of the orbital elements semimajor axis, eccentricity and inclination

4. to compare the qualitative and quantitative behavior of the proper elements with the respective r.m.s.

\section{The Computations}

The asteroids of table 1 were integrated over a time interval of $10^{8}$ years whereby the equations of motion have been computed by means of the Lie integration method (cf. Lichtenegger, 1984, Hanslmeier \& Dvorak, 1984). The outer solar system (i.e., Sun and the planets Jupiter through Neptune) has been used as dynamical model, where the Sun's mass has been increased by the masses of the inner planets in order to approximate the perturbations by the inner planets; relativistic terms were not taken into account.

From the whole time interval of 100 million years we determined 11 time intervals, where each covers 1 million years. These "short-time intervals" are defined as follows:

$$
I_{k}=[k,(k+1)] \text { Myrs with } k=0,10,20, \ldots, 100
$$

${ }^{2}$ Although the notation of ASCs is not accepted by all scientists it seems - at least for the authors that it hits the important points of indicating chaos via the relatively large positive Lyapunov exponent on one hand, and being stable for more than 1000 times the Lyapunov time on the other hand.

${ }^{3}$ The root mean square is defined as the square root of the mean or average of the square of an argument, i.e. $\sqrt{E\left[(x-m)^{2}\right]}$ with $m=E(x)$. 
TABLE I

Trojan asteroids in stable chaotic motion (cf. Milani et al., 1997).

\begin{tabular}{l|c|c|c|c|c}
\hline Asteroid & $a_{i n i}$ & $e_{\text {ini }}$ & $i_{i n i}$ & LCE $\times 10^{-5}$ & LT [ 10 $\left.0^{3} \mathrm{yrs}\right]$ \\
\hline (1868) Thersites & 5.290 & 0.110 & 16.8 & 1.12 & 89 \\
(1869) Philoctetes & 5.303 & 0.065 & 4.0 & 1.49 & 67 \\
1988 AK & 5.305 & 0.064 & 22.1 & 1.07 & 93 \\
(4543) Phoinix & 5.082 & 0.098 & 14.7 & 1.11 & 90 \\
4523 P-L & 5.236 & 0.048 & 0.9 & 2.12 & 47 \\
5187 T-2 & 5.131 & 0.031 & 8.6 & 1.24 & 81 \\
1991 HN & 5.098 & 0.011 & 8.3 & 1.73 & 58 \\
\hline (1173) Anchises & 5.326 & 0.137 & 6.9 & 2.04 & 49 \\
(2594) Acamas & 5.113 & 0.086 & 5.5 & 2.90 & 34 \\
(3451) Mentor & 5.086 & 0.070 & 24.7 & 1.90 & 53 \\
(5144) Achates & 5.232 & 0.273 & 8.9 & 1.10 & 91 \\
1988 RN11 & 5.269 & 0.096 & 1.4 & 1.99 & 50 \\
1989 UX5 & 5.104 & 0.024 & 4.3 & 6.38 & 16 \\
\hline
\end{tabular}

The evolution of the orbits within these 11 intervals was studied in a first step by the computations of the r.m.s. of the semimajor axes $(a)$, the eccentricities $(e)$ and the inclinations $(i)$.

In a second step we calculated the proper elements $d$ (the libration amplitude of the semi-major axis, i.e. $a-a_{J}=d \sin \theta+\ldots$, where $\theta$ is the argument of libration), $D$ (the libration amplitude of the critical argument, i.e. $\lambda-\lambda_{J}=\chi+D \cos \theta+$ ..., where $\chi$ denotes the position of the libration point), $e_{p}$ (the proper eccentricity was derived from the nonsingular variables $h=e \sin \varpi$ and $k=e \cos \varpi$ ) and $\sin \left(I_{p}\right)$ (the proper inclination was derived from the nonsingular variables $p=$ $\sin \left(I_{p} / 2\right) \sin \Omega$ and $q=\cos \left(I_{p} / 2\right) \sin \Omega$ ). Since proper elements are known as "quasi invariants", we checked their values within these eleven intervals defined above (each covers 1 million years). For their determination we used a numerical method similar to the one by Milani (1993, p. 61ff):

- The short periodic terms were eliminated using the method of Labrouste (cf. Bien \& Schubart, 1987 and Burger, 1998).

- The long periodic terms were determined with the aid of a numerical frequency analysis (=NFA) provided by Chapront (1995) and substracted from the signal of $h$ and $k$ for the proper eccentricities respectively of $p$ and $q$ for the proper inclinations.

- Using again the NFA we derived the proper elements $e_{p}$ and $\sin I_{p}$ with the two frequencies in the order of $300 " / y$ (for the eccentricities) and $10^{\prime \prime} / y$ (for the inclinations) depending on the asteroid.

Milani in his articles already determined proper elements for all - in those days available - Trojans with reliable orbits. Fig. 1 shows the agreement between the 


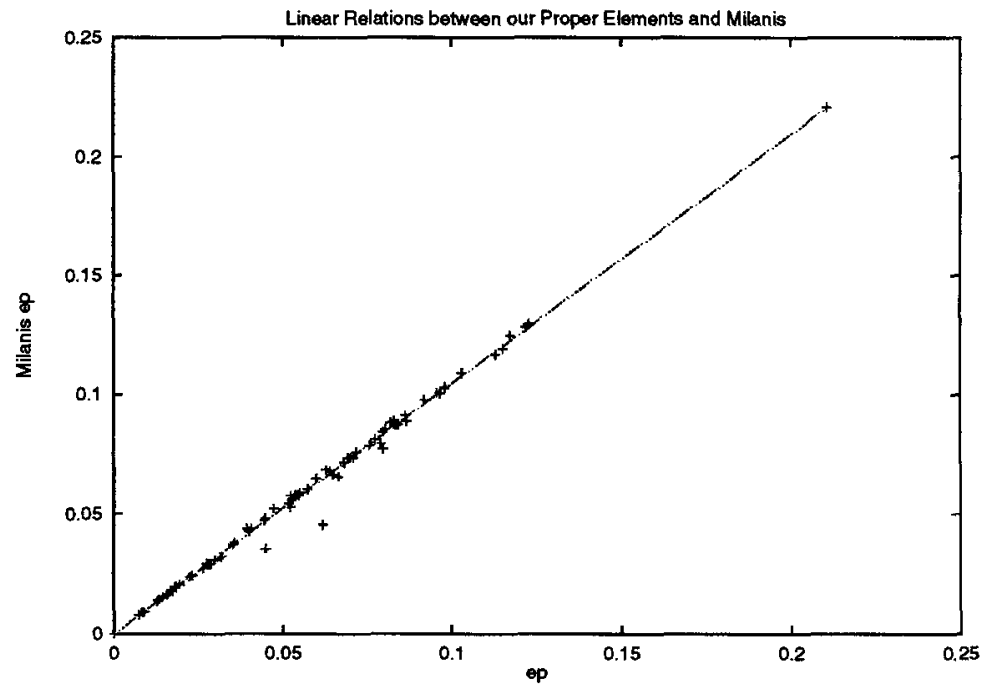

Fig. 1. Correlation between our proper eccentricities $(x-a x i s)$ and the values determined by Milani $(y-a x i s)$

two determinations for the proper elements $e_{p}$.

Additionally in table 2 we show the proper elements for our sample of 13 Trojans determined for the first million years ( $1^{\text {st }}$ interval of time). For Achates it was not possible to determine a proper $\sin I_{p}$ because of the very large variations in inclination (compare figure $4 \mathrm{~b}$ ).

\section{Long Term Orbital Evolution}

In a numerical study of Levison et al. (loc.cit) 21 of the 36 real Trojans turned out to be unstable in less than 4 billion years. In fig. 2 we show a similar plot as it is given by Levison et al. where all known Trojan asteroids are plotted in the plane of the proper elements $\mathrm{D}$ (i.e. libration amplitude) and $e_{p}$ (i.e. proper eccentricity). The full squares represent the asteroids of table 1 and the dotted line is Rabe's stability curve (cf. Rabe 1965) - which was determined in the simple model of the circular restricted three body problem. Shoemaker et al. (1989) concluded from the existence of objects above this stability curve that the true stability curve must be above Rabe's. In the study of Levison et al. the stable region exceeds Rabe's stability curve slightly, nevertheless there are still Trojans above Levison's stability boundary - like the asteroid Achates. Fig. 2 shows that 12 ASCs within Rabe's stability boundary have in general large D values $\left(D>20^{\circ}\right)$ and small proper eccentricities $\left(e_{p}<0.1\right)$. But no conclusion in what concerns their short Lyapunov time may be drawn from that.

Figs. 3 and 4 show the dynamical behaviour of 4 selected asteroids of the 
TABLE II

Proper elements for the $1^{\text {st }}$ time interval:

\begin{tabular}{|l|c|c|c|c|}
\hline Asteroid & $d$ & $D$ & $e_{p}$ & $\sin I_{p}$ \\
\hline (1868) Thersites & 0.1189 & 24.80 & 0.929 & 0.2850 \\
(1869) Philoctetes & 0.1112 & 22.16 & 0.506 & 0.0618 \\
1988 AK & 0.1085 & 22.79 & 0.0226 & 0.3737 \\
(4543) Phoinix & 0.1375 & 28.26 & 0.0455 & 0.2594 \\
4523 P-L & 0.1302 & 25.89 & 0.0637 & 0.0379 \\
5187 T-2 & 0.1089 & 21.99 & 0.0266 & 0.1758 \\
1991 HN & 0.1443 & 29.14 & 0.0270 & 0.1076 \\
\hline (1173)Anchises & 0.1281 & 25.39 & 0.0 .874 & 0.1374 \\
(2594) Acamas & 0.1673 & 33.543 & 0.0546 & 0.0950 \\
(3451) Mentor & 0.1345 & 28.76 & 0.0239 & 0.3983 \\
(5144) Achates & 0.0677 & 13.23 & 0.2175 & - \\
1988 RN11 & 0.1254 & 24.90 & 0.21275 & 0.0464 \\
1989 UX5 & 0.1536 & 31.04 & 0.1053 & 0.0481 \\
\hline
\end{tabular}

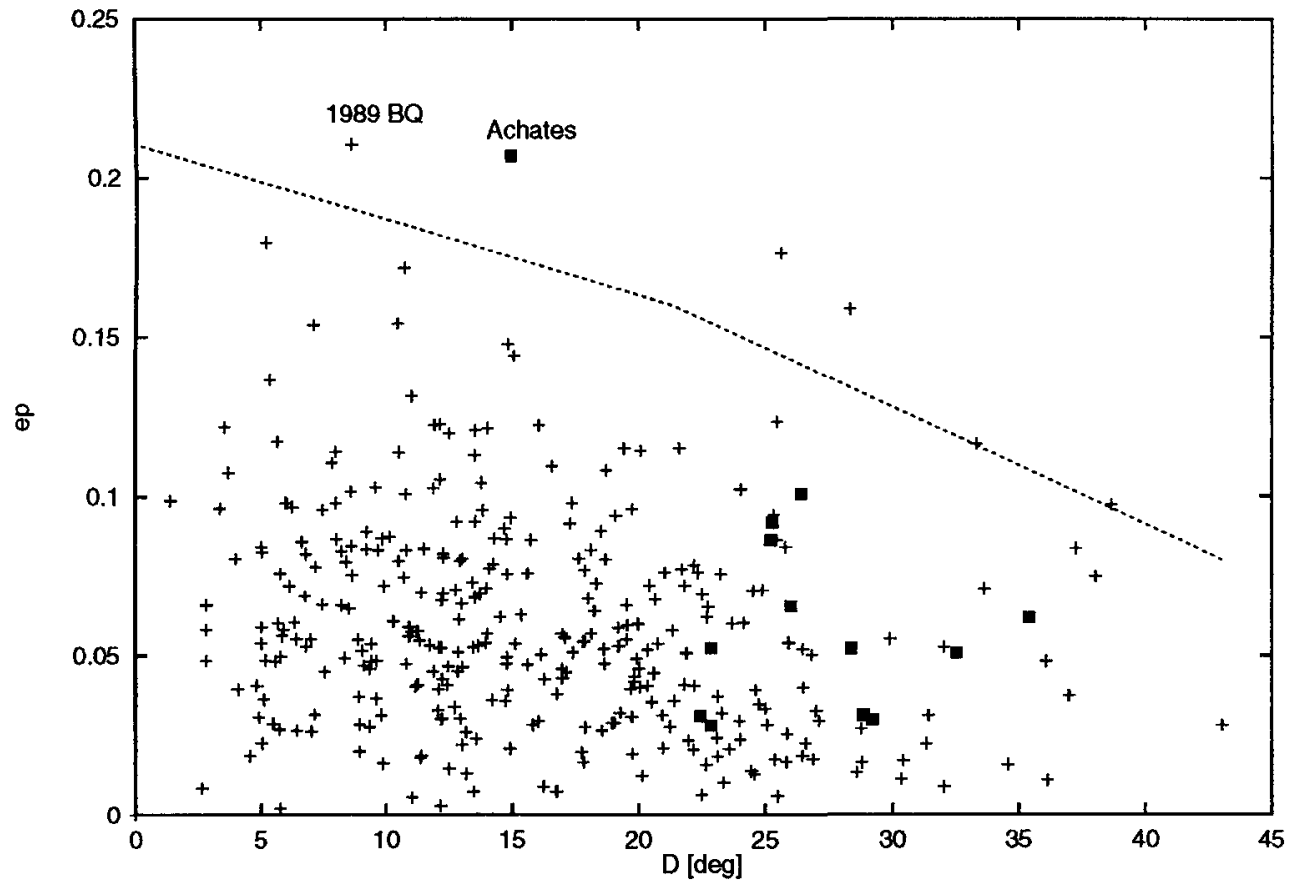

Fig. 2. All known Trojan asteroids in the proper elements plane $\left(D, e_{p}\right)$. The proper elements were calculated over 10 million years. The full squares represent the asteroids of table 1 and the dotted line is Rabe's stability curve (cf. Rabe 1965). 

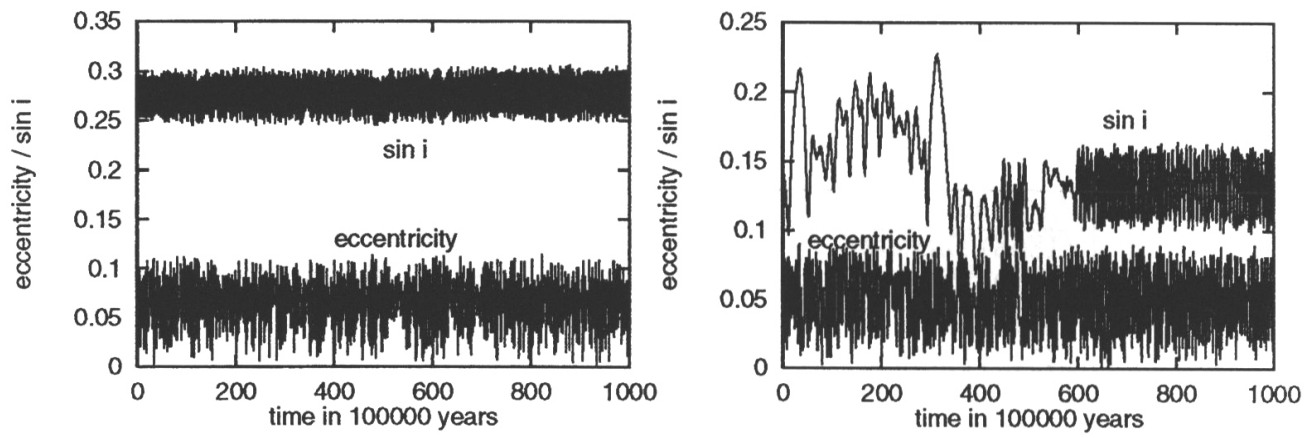

Fig. 3. Orbital evolution for 100 Myrs of two $L_{4}$ ASCs: (4543) Phoinix with $e_{0}=0.098$ and $i_{0}=14.7$ (left) and $1991 \mathrm{HN}$ with $e_{0}=0.011$ and $i_{0}=8.3$ (right). Note that on the $y$-axis we plotted the eccentricity and the sine of the inclination.
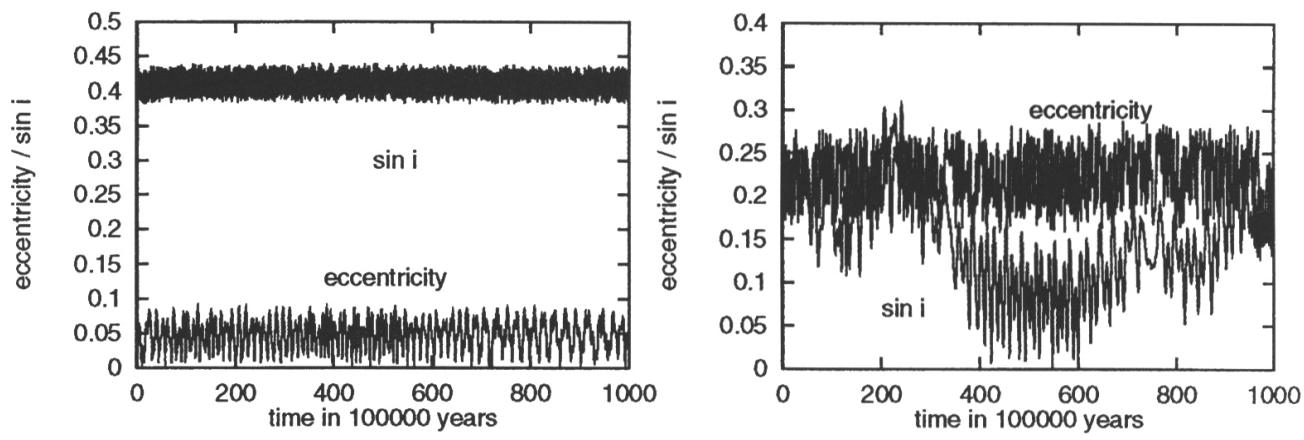

Fig. 4. Orbital evolution for 100 Myrs of two $L_{5}$ ASCs: (3451) Mentor with $e_{0}=0.07$ and $i_{0}=24.7$ (left) and (5144) Achates with $e_{0}=0.273$ and $i_{0}=8.9$ (right). Note that on the $y$-axis we plotted the eccentricity and the sine of the inclination.

Trojan ASCs for the whole time interval of 100 Million years. The evolution of eccentricity and inclination does not show significant changes over the whole time interval for the $L_{4}$ asteroid Phoinix and the $L_{5}$ asteroid Mentor. The dynamical evolution is quite different for $1991 \mathrm{HN}$ and for Achates: while the first one - an $L_{4}$ asteroid - is suffering strong and irregular perturbations of the inclination for the first 60 million years and then it is in a very regular looking orbit the second one - an $L_{5}$ asteroid - shows strong variations in the inclination from 30 million years on. Both effects may be caused by second order resonances inside the $1: 1$ resonance with Jupiter; this will be studied more detailed in connection with the interesting escape of the asteroid Thersites, which escaped after about 26 million years. Therefore, we excluded this Trojan from our numerical analysis. 


\section{Comparison of the Proper Elements with the r.m.s.}

In fig. 5 the proper elements $e_{p}$ are compared to the r.m.s.(e) for the same 11 intervals; one recognizes how well the two curves agree. The correlation coefficients are 0.993 for the $L_{4}$ and 0.978 for the $L_{5}$ asteroids. A comparison of the proper element $\sin I_{p}$ with the r.m.s. of the inclinations for the time intervals does not show such an agreement. In fig. 6 we show two examples namely the $L_{4}$ Trojan $88 \mathrm{AK}$ and the $L_{5}$ Trojan $88 \mathrm{RN} 11$. The calculated correlation coefficients of 0.883 and 0.693 are small and thus these two quantities are obviously not correlated. The similar behavior of the r.m.s.(a) and the proper element $d$ is obvious through the pragmatic definition of $d$.

In the former mentioned paper by Levison et al. (loc.cit.) fig. 2 shows the variations of $\mathrm{D}$ and $e_{p}$ for a "typical Trojan" in an unstable orbit over a time scale of $1.5 \times 10^{9}$ years. The changes of the proper eccentricity are in the same order of magnitude in their example $\left(0.06<e_{p}<0.08\right)$ as in our calculations for the ASCs (e.g. for Achates we found the following variations: $0.217<e_{p}<0.226$ ).

\section{Conclusions}

In this numerical investigation we examined the long term stability of a sample of 13 especially selected Trojans with relatively small Lyapunov times covering a time interval of 100 million years.

The results can be summarized as follows:

- the proper elements are slowly varying quantities for most of the asteroids examined, with the exception of $1991 \mathrm{HN}$ and Achates (large variations of the inclination)

- the asteroid Thersites escaped after 26 million years

- the proper elements $e_{P}$ are correlated with the corresponding r.m.s. of the eccentricities and thus the r.m.s.(e) may - as a first rough estimation - serve as proper element indicator for $e_{p}$; also the r.m.s.(a) may serve as proper element indicator for $d$

- the proper element $\sin I_{p}$ is seemingly not correlated with the r.m.s. of the inclination.

In summary no special dynamical evolution for these asteroids has been found with the exception of Thersites. So it remains an unsolved problem why these asteroids have such short Lyapunov times; more work has to be done to answer this interesting question. 

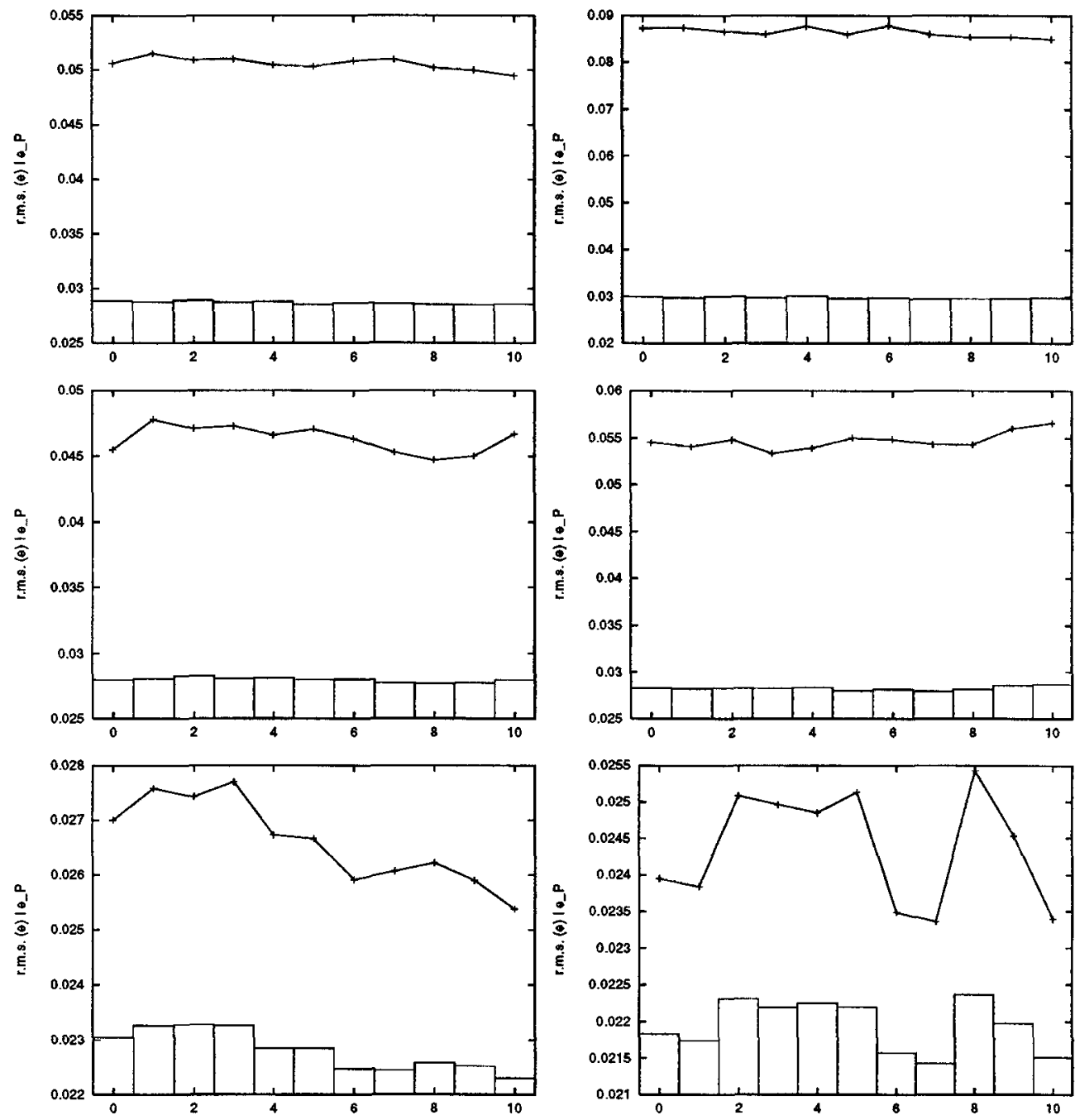

Fig. 5. r.m.s.(e) (boxes) and proper element $e_{p}$ (lines) versus 11 different time intervals of $\Delta t=1$ Million years: $[0,1],[10,11], \ldots[100,101]$ for the $L_{4}$ Trojans: Philoctetes (upper left), Phoinix (middle left), $1991 \mathrm{HN}$ (lower left) and for the $L_{5}$ Trojans: Anchises (upper right), Acamas (middle right) and Mentor (lower right). 

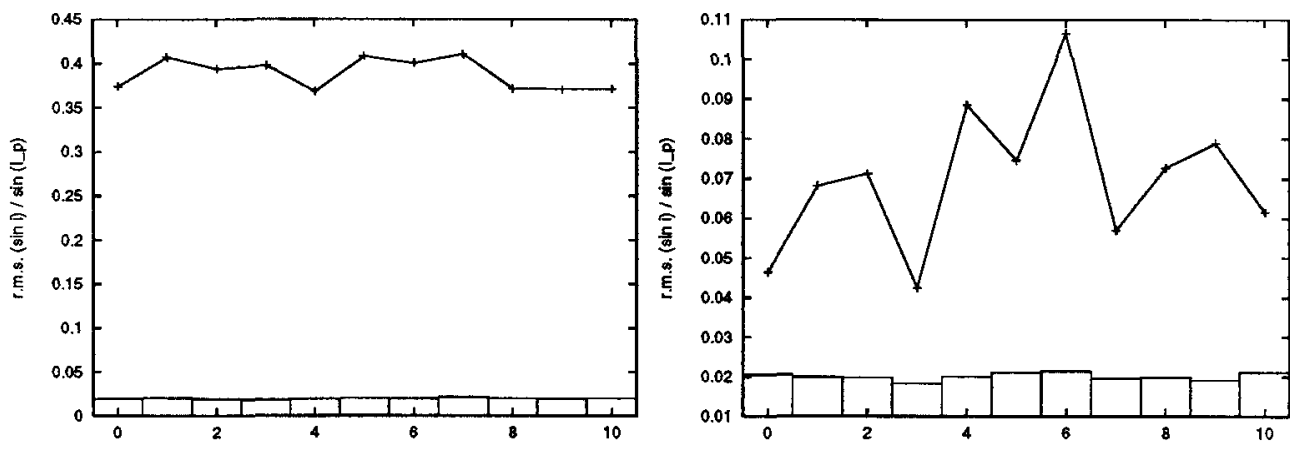

Fig. 6. r.m.s.(i) (boxes) and proper element $\sin I_{p}$ (lines) versus 11 different time intervals of $\Delta t=1$ Million years: $[0,1],[10,11], \ldots[100,101]$ for the $L_{4}$ Trojan $88 \mathrm{AK}$ (left) and for the $L_{5}$ Trojan: 88RN11(right)

\section{Acknowledgements}

We are grateful to the critical comments by Dr. Morbidelli and also by Dr. FerrazMello who helped to improve the paper significantly. Special thanks go to Dr. Wodnar for many discussions on the problem of proper elements.

The work presented here was carried out within the framework of the Jubiläumsfondsprojekt Nr. 6446 of the Österreichische Nationalbank. Two of the authors (EPL and CB) wish to thank Prof. J. Henrard for the financial support. EP-L received a travel grant from the Österreichische Forschungsgemeinschaft.

\section{References}

Barber, G.: 1986, “The Orbits of Trojan Asteroids”, in C.I. Lagerkvist, B.A. Lindblad, H. Lundstedt and H. Rickman (eds.), Asteroids, Comets, Meteors II, University of Uppsala, 161.

Bien, R., Schubart, J.: 1984, “Trojan orbits in secular resonances", Cel.Mech \& Dyn. Astro., 34, 425.

Bien, R., Schubart, J.: 1987, “Three characteristic parameters for the Trojan group of asteroids", Astron.Astrophys., 175, 292.

Burger Ch.: 1998, "The Method of Labrouste with an Application in Celestial Mechanics", Diploma thesis, University of Vienna, $75 \mathrm{p}$.

Chapront, J.: 1995, "Representation of planetary ephemerides by frequency analysis. Application to the five outer planets", Astron. Astrophys., 109, 181.

Dvorak, R., Pilat-Lohinger, E.: 1998, "Les astéroides troyens: une nouvelle analyse" in D. Benest \& C. Froeschlé (eds.) Aux Frontières de la Dynamique chaotique des systèmes gravitationelles O.C.A. Observatoire de Nice, 19.

Erdi, B.: 1984, "Critical inclination of Trojan asteroids", Cel.Mech \& Dyn. Astro., 34, 435.

Erdi, B.: 1988, “ Long periodic perturbations of Trojan asteroids", Cel.Mech. \& Dyn. Astro., 43, 303.

Erdi, B.: 1996, "On the Dynamics of Trojan Asteroids", in S. Ferraz-Mello, B. Morando, and J.E. Arlot (eds.), IAU Symposium 172, Dynamics, Ephemerides and Astrometry in the Solar System, 171 .

Erdi, B.:1997, “The Trojan Problem”, Cel.Mech \& Dyn. Astro., 65, 149.

Hansimeier, A., Dvorak, R.: 1984, "Numerical Integration with Lie Series", Astron. Astrophys., 132, 203. 
Lemaitre, A.: 1993, "Proper Elements: What are they", Cel.Mech. \& Dyn. Astro., 56, 103.

Levison, H. F., Shoemaker, E.M. and Shoemaker C.S.: 1997, "Dynamical Evolution of Jupiter's Trojan asteroids",Nature, $385,42$.

Lichtenegger, H.: 1984, "The Dynamics of Bodies with Variable Masses", Cel.Mech. 34, p. 357.

Milani, A.: 1993, "The Trojan Asteroid Belt: Proper Elements, Stability, Chaos and Families", Cel.Mech \& Dyn. Astro., 57, 59.

Milani, A.: 1994, "The Dynamics of the Trojan Asteroids", in A. Milani, M. di Martino, A. Cellino (eds.) IAU Symposium 160, ACM 1993, Kluwer Academic Publishers, The Netherlands. 159.

Milani, A., Nobili, A., Kneževic, Z.: 1997, "Stable Chaos in the Asteroid Belt", Icarus, 125, 13.

Pilat-Lohinger E., Dvorak, R., Burger Ch.: 1998, "Asteroids in stable chaotic motion" in R. Dvorak, H.Haupt and K. Wodnar (eds.): Modern Astrometry and Astrodynamics, Verlag der Österreich.Akad.d.Wiss., Wien, 29.

Rabe, J.: 1965, “Limiting Eccentricities for Stable Trojan Librations", Astron. J., 70, 687.

Schubart, J., Bien, R.: 1984, "An application of Labrouste's method to quasi-periodic asteroidal motion", Cel.Mech \& Dyn. Astro., 34, 443.

Schubart, J., Bien, R.: 1987, "Trojan asteroids - Relations between dynamical parameters ", Astron. Astrophys., 175, 200.

Shoemaker, E.M., Shoemaker, C.S., Wolfe, R.F.: 1989, "Trojan asteroids - Population, dynamical structure and origin of the L4 and L5 swarms." in Asteroids II, Tucson, Univ. of Arizona Press, 487 